\title{
Measuring Temperature-Dependent Miscibility for Polymer Solar Cell Blends: An Easily Accessible Optical Method Reveals Complex Behavior
}

\author{
Zhengxing Peng, ${ }^{\dagger}$ Xuechen Jiao, ${ }^{\dagger}$ Long Ye, ${ }^{\dagger}$ Sunsun Li, ${ }^{\ddagger}$ Jeromy James Rech, ${ }^{\S}$ Wei You, ${ }^{\S}$ \\ Jianhui Hou, ${ }^{\ddagger \odot}$ and Harald Ade ${ }^{*}+\odot$ \\ ${ }^{\dagger}$ Department of Physics and Organic and Carbon Electronics Lab (ORaCEL), North Carolina State University, Raleigh, North \\ Carolina 27695, United States \\ ${ }^{\ddagger}$ State Key Laboratory of Polymer Physics and Chemistry, Beijing National Laboratory for Molecular Sciences, Institute of Chemistry, \\ Chinese Academy of Sciences, Beijing 100190, China \\ ${ }^{\S}$ Department of Chemistry, University of North Carolina at Chapel Hill, Chapel Hill, North Carolina 27599, United States
}

\begin{abstract}
In bulk-heterojunction polymer solar cells (PSC), the molecular-level mixing between conjugated polymer donors and small-molecule acceptors plays a crucial role in obtaining a desirable morphology and good device stability. It has been recently shown that the thermodynamic limit of this mixing can be quantified by the liquidus miscibility, the composition of the small-molecule acceptor in amorphous phases in the presence of small-molecule crystals, and then converted to the FloryHuggins interaction parameter $\chi$. This conversion maps out the amorphous miscibility. Moreover, the quantitative relations between $\chi$ and the fill factor of PSC devices were established recently. However, the commonly used measurement of this liquidus miscibility, scanning transmission X-ray microscopy, is not easily and readily accessible. Here, we delineate a method based on common visible light microscopy and ultraviolet-visible absorption spectroscopy to replace the X-ray measurements. To demonstrate the feasibility of this technique and methodology, a

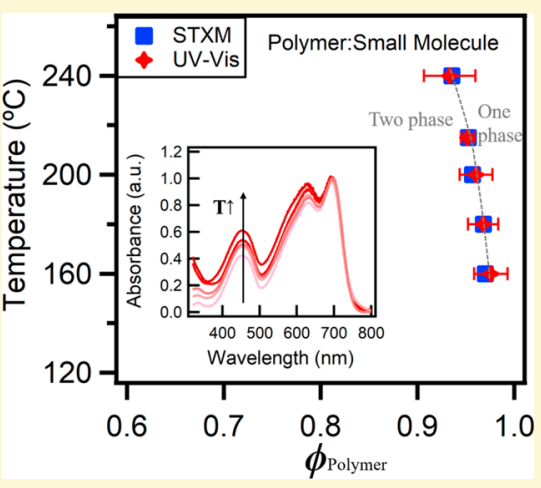
variety of conjugated polymers (PffBT4T- $\mathrm{C}_{9} \mathrm{C}_{13}$, PDPP3T PBDT-TS1, PTB7-Th, and FTAZ) and their miscibility with fullerenes or nonfullerene small molecules $\left(\mathrm{PC}_{71} \mathrm{BM}, \mathrm{PC}_{61} \mathrm{BM}\right.$, and $\left.\mathrm{EH}-\mathrm{IDTBR}\right)$ are characterized. The establishment of this methodology will pave the way to a wider use of the liquidus miscibility and the critical miscibility-function relations to optimize the device performance and obtain good stability in PSCs and other devices.
\end{abstract}

\section{INTRODUCTION}

The strategy of blending two or more materials, bringing about synergistic and surprising properties in structure, electronics, dielectrics, optics, thermodynamics, mechanics, and texture, contributes much to the development of organic solar cells (OSC), ${ }^{1}$ organic light-emitting diodes (OLED), ${ }^{2}$ organic fieldeffect transistors (OFET), ${ }^{3}$ ferroelectric devices, ${ }^{4}$ and organic thermoelectrics. ${ }^{5}$ A fundamental thermodynamic aspect about blending is how much the blended materials can mix with each other at the molecular level. This is referred to as miscibility, a parameter that is often significant in determining final properties of applications. For example, in thermoelectrics, the miscibility between the host and dopant materials determines the dopant concentration, which may affect the electrical conductivity. ${ }^{5}$ Particularly in the bulk heterojunction (BHJ) polymer solar cells (PSCs), the miscibility between electron-donating polymers and electron-accepting molecules in the photoactive layers plays an important role in the morphology formation, ${ }^{6-10}$ which has a direct impact on the exciton dissociation, charge transport, and charge recombination. The molecular mixing is an intrinsic and defining property, whose absence provides the driving force for mixed donor and acceptor materials to phase separate. Quantifying and measuring this miscibility is necessary and critical for understanding polymer solar cells and the ability of new materials to combine to achieve high performance. Furthermore, creating a wide-ranging database will be also help in developing predictive computational tools.

In polymer solar cells, amorphous miscibility between the donor and the acceptor is important for device performance for several reasons. First, molecular mixing governs phase behavior and morphology formation and, consequently, affects charge recombination and charge collection efficiency, which will directly influence device performance. ${ }^{6,7}$ Second, amorphous miscibility has an impact on some measured morphological parameters, such as long period and root-mean-square composition variations related to average purity, ${ }^{11}$ whose quantitative relations to short-circuit current $\left(J_{\text {sc }}\right)$ or fill factor (FF) have been established in a number of systems. ${ }^{12,13}$ Recently,

Received: February 28, 2018

Revised: $\quad$ May 21, 2018

Published: May 23, 2018 
the approach to converting liquidus miscibility, which quantifies the composition of the mixed, amorphous domains in local thermodynamic equilibrium in the presence of small-molecule crystals, into Flory-Huggins interaction parameter $\chi(T)$ has been introduced and the quantitative relations between $\chi(T)$ and FF have been reported. ${ }^{14}$ Moreover, miscibility is also a principal factor to determine the upper limit of the purity in the mixed phases, $^{11}$ and changes the sensitivity of domain spacing to fullerene loading. ${ }^{15}$ Third, the amorphous miscibility can also determine the stability of active layers and excessively low miscibility may induce burn-in degradation. ${ }^{16}$ There exists an optimum polymer-small molecule miscibility in the active layer. For example, for strongly immiscible systems, large-scale phase separation or excessive purification will occur which will prevent the polymer donors from establishing the desirable nanoscale morphology, but, for highly miscible systems, charge recombination will be intensified because the small molecule concentration is much higher than the percolation threshold ${ }^{17,18}$ in the mixed phase. The ideal miscibility is likely near the percolation threshold. The measurement of the temperaturedependent liquidus miscibility can help map out the phase diagram, giving us a better understanding of the thermodynamic properties of a certain system.

Previously, liquidus miscibility has been successfully measured by several methods, such as scanning transmission X-ray microscopy (STXM), $6,11,15,18$ dynamic secondary ion mass spectrometry (DSIMS), ${ }^{7,19}$ and energy filtered transmission electron microscopy (EFTEM). ${ }^{20-22}$ Among these, STXM has been the method used most extensively. However, STXM requires the use of a synchrotron radiation facility, which is not easily accessible for all the research groups or at any time. DSIMS can measure liquidus and even amorphous miscibility ${ }^{14}$ straightforwardly if there is a unique molecular fragment. Unfortunately, it is expensive and often unavailable in some laboratories that focus on organic electronics. Additionally, some systems require deuteration to provide a discriminating signal. It also requires a high level of expertise in the sample preparation of suitable bilayers. EFTEM is not a general method for liquidus miscibility measurement for the reason that it is not sensitive enough to differentiate two mixed organic materials with low material contrast. $^{21,22}$ The limitation of these methods and the challenges to using them routinely hinders the universal determination and application of liquidus miscibility to a wider scope.

Here, we present an easily and widely accessible method to measure the uncorrected liquidus miscibility, a measure that has not yet normalized out the crystalline volume fraction of a semicrystalline polymer. It is based on a combination of visible light microscopy (VLM) and standard ultraviolet-visible (UVvis) absorption spectroscopy without any spatial resolution. Both methods are readily available in many research laboratories. This UV-vis method uses similar sample preparation protocols to measurements using STXM. After reaching local thermodynamic equilibrium, the absorption spectra of the mixed amorphous domains in blend films is a linear combination of the reference spectra of its two constituent components, which can be measured with neat films. By fitting the blend spectra with a linear superposition of the neat reference spectra, the (uncorrected) liquidus miscibility is obtained. Starting with PffBT4T- $\mathrm{C}_{9} \mathrm{C}_{13}: \mathrm{PC}_{71} \mathrm{BM}$ as a model system, whose uncorrected liquidus miscibility has previously been characterized, ${ }^{16}$ we extend the approach to characterizing other systems including PDPP3T:PC ${ }_{61}$ BM, PBDT-TS1:PC ${ }_{71}$ BM, PTB7-Th:PC ${ }_{71} B M$, and FTAZ:EH-IDTBR. These five combinations are representatives of a broad range of different types of materials systems, including a nonfullerene acceptor system and yield high performing devices with significant interest to the research community. The results from this UV-vis method are successfully verified by STXM method. This new and easily accessible method is of significance for the wide use of liquidus miscibility in helping optimize polymer solar cells and other devices.

\section{EXPERIMENTAL SECTIONS}

2.1. Materials. The polymer PBDT-TS $1^{23}$ and $F T A Z^{24}$ used in this work are synthesized according to previously published methods. The PTB7-Th and the PDPP3T are purchased from Solarmer, Inc.; the PffBT4T- $\mathrm{C}_{9} \mathrm{C}_{13}$ is obtained from Raynergy Tek, and the EH-IDTBR is acquired from 1-Material.

2.2. Instruments. Transmission VLM images are acquired using a Nikon Labophot-2 microscope. The UV-vis absorption spectra are recorded using a Cary 50 spectrophotometer with the wavelength from 300 to $1000 \mathrm{~nm}$. In the UV-vis experiment, optical density (O.D.) is evaluated by measuring the transmission intensity with the active layer $(I)$ and without the active layer $\left(I_{0}\right)$ and calculated as O.D. $=-\log _{10}\left(I / I_{0}\right)$. STXM measurements are conducted at beamline 5.3.2.2 $2^{25}$ of the Advanced Light Source (ALS) using films floated onto TEM grids and analyzed following previously established protocols. ${ }^{26}$

2.3. Experimental Procedures. First, all the glass substrates are cleaned via ultrasonication in deionized water, acetone, and isopropanol for $15 \mathrm{~min}$, respectively, and then cleaned with ultraviolet ozone light for another $15 \mathrm{~min}$. For the model system, PffBT4T- $\mathrm{C}_{9} \mathrm{C}_{13}: \mathrm{PC}_{71} \mathrm{BM}$, the solution for blend films are prepared by dissolving $15 \mathrm{mg} / \mathrm{mL}$ PffBT4T- $\mathrm{C}_{9} \mathrm{C}_{13}$ and $15 \mathrm{mg} /$ $\mathrm{mL} \mathrm{PC}_{71} \mathrm{BM}$ in 1,2-dichlorobenzene, while the solutions for the neat polymer films and neat fullerene films contain $15 \mathrm{mg} / \mathrm{mL}$ PffBT4T- $\mathrm{C}_{9} \mathrm{C}_{13}$ and $15 \mathrm{mg} / \mathrm{mL} \mathrm{PC}_{71} \mathrm{BM}$, respectively. The films are made by spin-casting the solutions at $1000 \mathrm{rpm}$ onto polystyrenesulfonate (PSS) coated glass substrates after stirring the solutions at $80{ }^{\circ} \mathrm{C}$ overnight. The parameters for other systems are described in the Supporting Information. Second, PffBT4T- $\mathrm{C}_{9} \mathrm{C}_{13}: \mathrm{PC}_{71} \mathrm{BM}$ blend films are isothermally annealed at various temperatures, such as $160^{\circ} \mathrm{C}, 180^{\circ} \mathrm{C}$, and so on, up to 3 days to reach the local thermodynamic equilibrium, verified by the VLM images displayed later. All thermal annealing is carried out inside a glovebox $\left(\mathrm{N}_{2}\right.$ atmosphere $)$ to avoid possible effects of photooxidative degradation at elevated temperatures that might occur under ambient conditions. Subsequently, the UVvis absorption spectra of the neat films and thermally annealed blend films are measured via a UV-vis absorption spectroscopy. Since the optical density (O.D.) follows the formula O.D. $=\mu \rho t$, where $\mu$ is the energy-dependent mass absorption coefficient, $\rho$ is the density, and $t$ is the film thickness, all the measured UV-vis absorption spectra of reference spectra should be normalized by its mass thickness $(\rho t)$ to yield the mass-absorption coefficient $\mu$. Finally, the spectrum of a blend film annealed at a certain temperature is fitted with a linear superposition of the reference spectra of neat films, with the ratio of linear coefficients giving the uncorrected liquidus miscibility at this temperature. We refer to average composition ratio as uncorrected liquidus miscibility since some of the polymers are semicrystalline and one would need to be normalized out the crystalline volume fraction of semicrystalline polymer to arrive at the correct composition of the mixed amorphous domains. After measuring the UV-vis absorption spectra, the blend films are floated onto deionized 


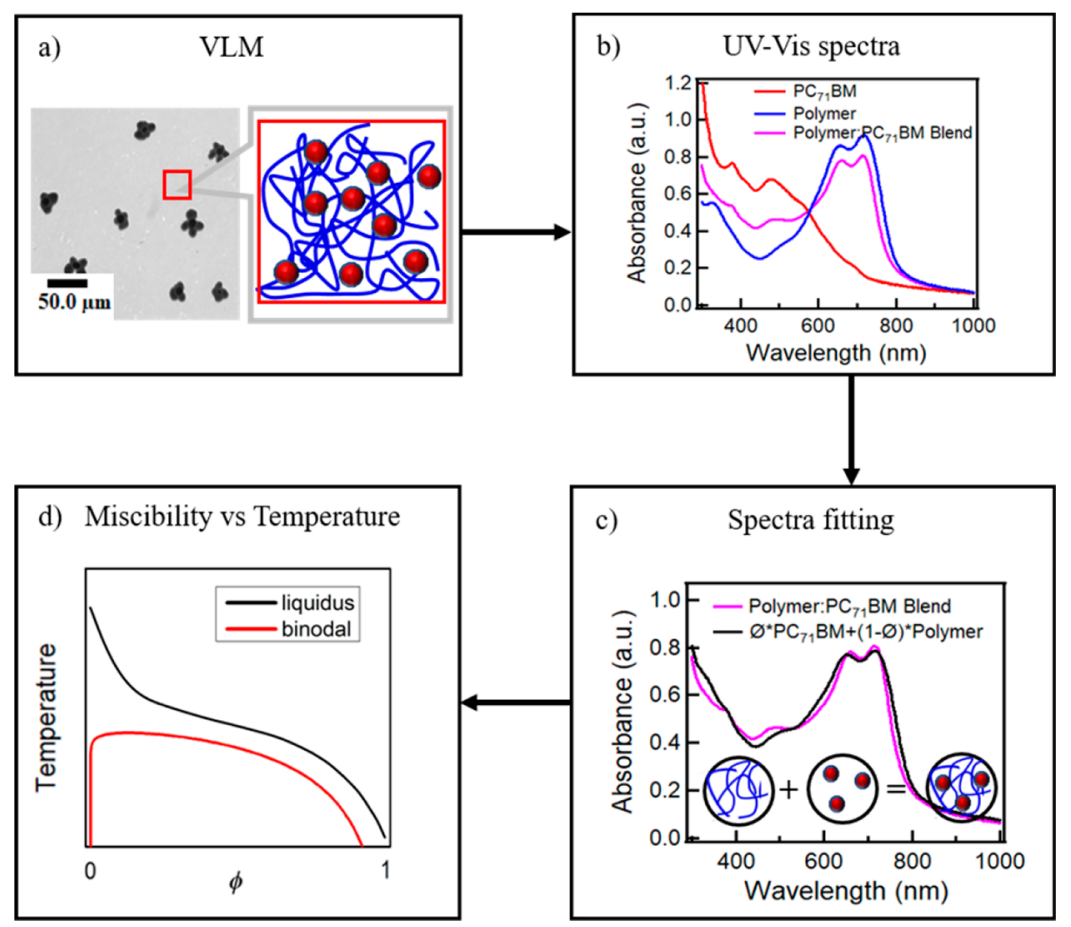

Figure 1. A flowchart describing the steps to measure the temperature-dependent miscibility. Take a polymer:PC $\mathrm{P}_{71} \mathrm{BM}$ system as an example. (a) Use of visible light microscopy (VLM) to ensure the local thermodynamic equilibrium is reached. This local thermodynamic equilibrium state is reached in a thermally annealed polymer:fullerene blend film, when the fullerene crystals are embedded in a uniform matrix without depletion gradients. (b) UV-vis absorption spectra are acquired on the neat films and thermally annealed blend films. (c) The polymer:fullerene blend spectrum is fitted with the linear combination of the neat, mass-thickness normalized reference spectra. (d) The liquidus line can be acquired. In the phase diagram, the liquidus is the equilibrium composition between the amorphous, mixed phase and the PCBM crystals corresponding to the liquidus miscibility while the binodal is the coexistence curve of binary amorphous mixtures of polymer:PCBM blends corresponding to the amorphous miscibility when PCBM crystals have been suppressed. We note that the UV-vis averages over both the polymer-rich matrix as well as the PCBM crystals. We will show later that the presence of PCBM crystals does not introduce a significant measurement error, as the PCBM crystals are so thick and absorbing that they act similar to any completely opaque "dust spec" and thus do not contribute significantly to the UV-vis spectrum or a heterogeneous sample measured in absorption by detecting transmitted light.

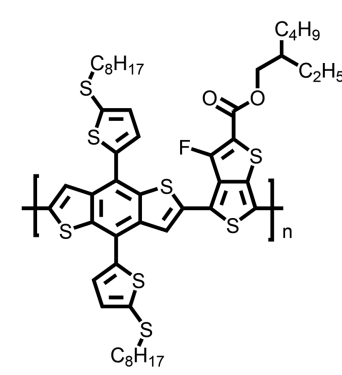

PBDT-TS1

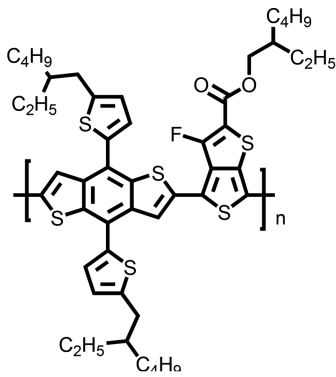

PTB7-Th

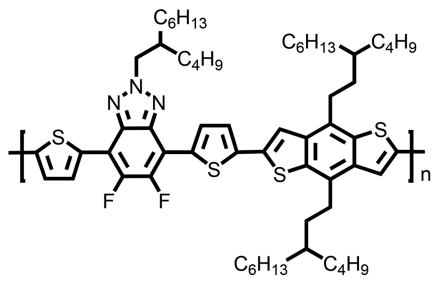

FTAZ

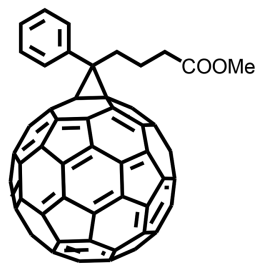

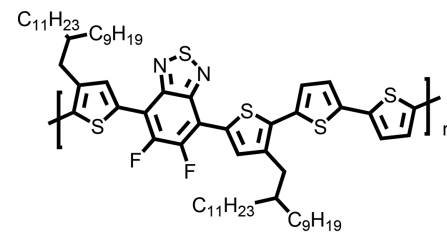

PffBT4T-C $\mathrm{C}_{9} \mathrm{C}_{13}$

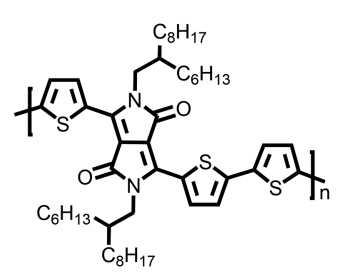

PDPP3T

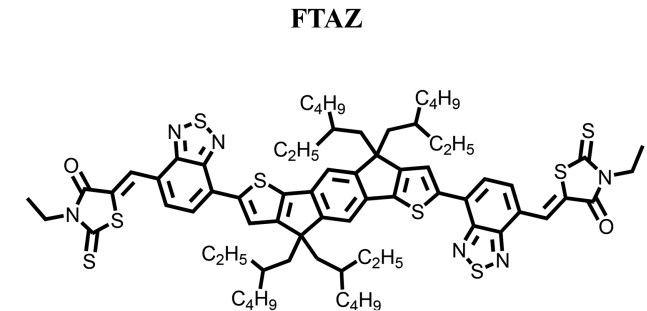

EH-IDTBR
$\mathrm{PC}_{61} \mathrm{BM}$

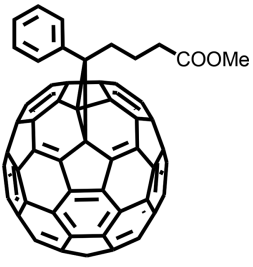

$\mathbf{P C}_{71} \mathbf{B M}$

Figure 2. Molecular structures of materials used.

water and transferred and mounted on TEM grids for later

STXM measurement to measure the uncorrected liquidus miscibility on the very same samples. The overall measuring flowchart is depicted in Figure 1.

\section{RESULTS}

To evaluate the capability of the UV-vis method in characterizing the (uncorrected) liquidus miscibility, we test the following five different systems that are representative of comprehensive OPV systems: PffBT4T- $\mathrm{C}_{9} \mathrm{C}_{13}: \mathrm{PC}_{71} \mathrm{BM}$, PDPP3T:PC ${ }_{61} \mathrm{BM}$, 
a)

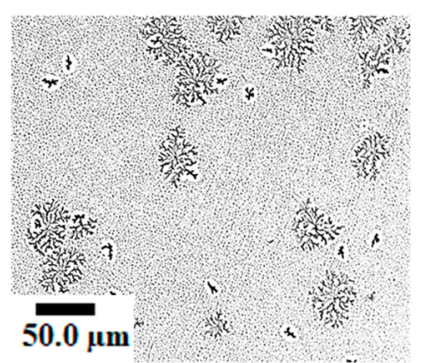

d)

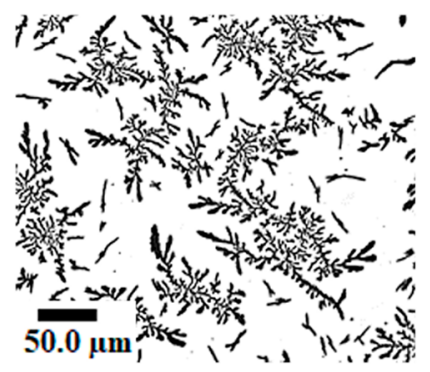

b)

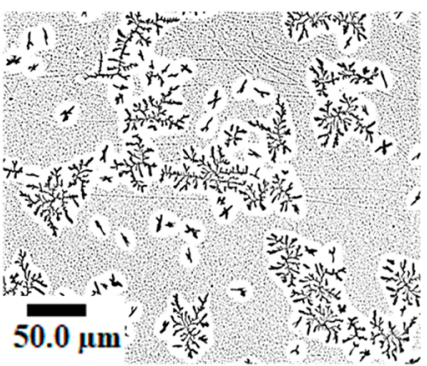

e)

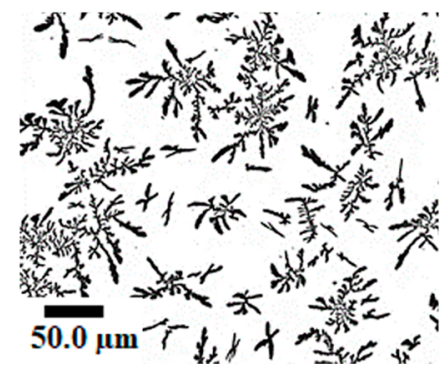

c)

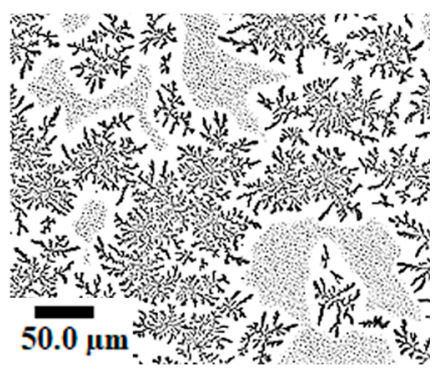

f)

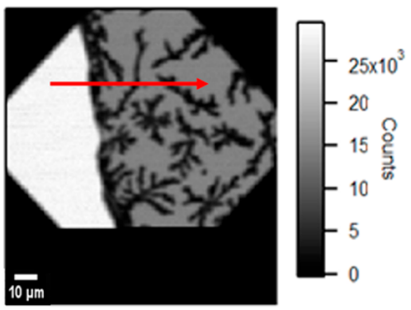

Figure 3. Transmission VLM images of phase-separated blends of a $6: 4(\mathrm{w} / \mathrm{w})$ PffBT4T- $\mathrm{C}_{9} \mathrm{C}_{13}: \mathrm{PC}_{71} \mathrm{BM}$ annealed at $240{ }^{\circ} \mathrm{C}$ for $(\mathrm{a}) 5 \mathrm{~min},(\mathrm{~b}) 15 \mathrm{~min}$, (c) $30 \mathrm{~min},(\mathrm{~d}) 23 \mathrm{~h}$, and (e) $80 \mathrm{~h}$. PCBM depletion regions are observed to be the small areas beside the PCBM crystals as shown in panel a and then they grow with annealing time displayed in panels $b$ and $c$ until they are expanded to the whole region in panels $d$ and e. (f) 2D STXM image of 6:4 (w/ w) PffBT4T- $\mathrm{C}_{9} \mathrm{C}_{13}: \mathrm{PC}_{71} \mathrm{BM}$ blend annealed at $200{ }^{\circ} \mathrm{C}$ for 3 days and the red arrow indicates the location and direction of STXM line scan. The uniform white area is a region without sample that is used to measure the incident X-ray intensity.

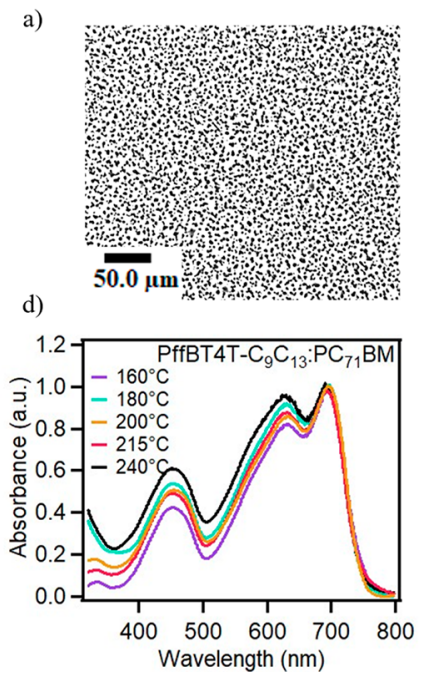

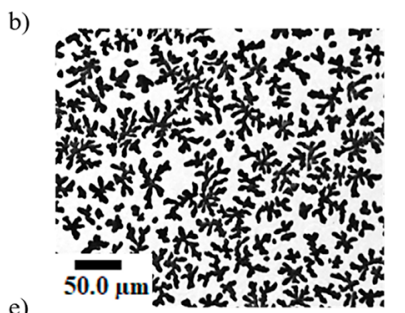

e)

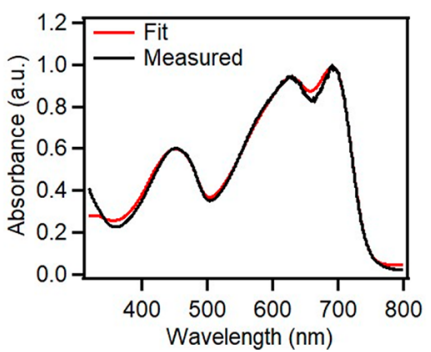

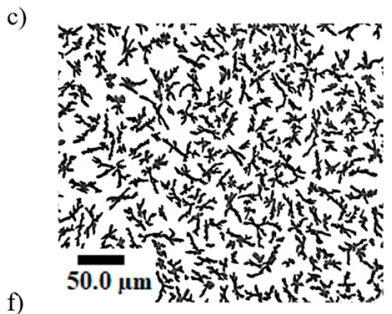

f)

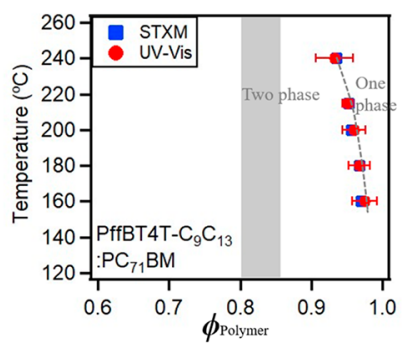

Figure 4. Transmission VLM images of phase-separated blends of $1: 1(\mathrm{w} / \mathrm{w})$ PffBT4T-C $\mathrm{C}_{9} \mathrm{C}_{13}: \mathrm{PC}_{71} \mathrm{BM}$ annealed at $(\mathrm{a}) 160{ }^{\circ} \mathrm{C}$ for $78 \mathrm{~h}$, $(\mathrm{b}) 180{ }^{\circ} \mathrm{C}$ for $65 \mathrm{~h}$, and (c) $20{ }^{\circ} \mathrm{C}$ for $78 \mathrm{~h}$. (d) UV-vis absorption spectra of 1:1 (w/w) PffBT4T- $\mathrm{C}_{9} \mathrm{C}_{13}: \mathrm{PC}_{71} \mathrm{BM}$ blends annealed at different temperatures and the highest intensity of each spectrum is normalized to be 1. (e) Comparison between the fitted spectrum from UV-vis method and the measured spectrum for 1:1 (w/w) PffBT4T- $\mathrm{C}_{9} \mathrm{C}_{13}: \mathrm{PC}_{71} \mathrm{BM}$ blend annealed at $240^{\circ} \mathrm{C}$. (f) The plot of temperature dependent uncorrected liquidus miscibility for PffBT4T$\mathrm{C}_{9} \mathrm{C}_{13}$ mixed with $\mathrm{PC}_{71} \mathrm{BM}$, where $\phi_{\text {Polymer }}$ is the uncorrected composition of PffBT4T- $\mathrm{C}_{9} \mathrm{C}_{13}$ in the amorphous, mixed phase. It is determined by both the UV-vis method and STXM. The gray band is the reported percolation threshold, ${ }^{8}$ where the polymer volume fraction is around $0.8-0.85$. The liquidus line separating one phase and two phase regions are conceptual and not fits to a model or theory.

PBDT-TS1:PC ${ }_{71}$ BM, PTB7-Th:PC ${ }_{71} \mathrm{BM}$, and FTAZ:EHIDTBR. The molecular structures of the materials used are shown in Figure 2. Among these, PffBT4T- $\mathrm{C}_{9} \mathrm{C}_{13}$ and PDPP3T are common semicrystalline polymers, while PBDT-TS1, PTB7Th, and FTAZ are common amorphous polymers. PffBT4T$\mathrm{C}_{9} \mathrm{C}_{13}: \mathrm{PC}_{71} \mathrm{BM}$, PDPP3T:PC ${ }_{61} \mathrm{BM}$, and FTAZ:EH-IDTBR are systems with relatively high Flory-Huggins interaction parameter $\chi$ and low miscibility while PBDT-TS1:PC ${ }_{71} \mathrm{BM}$ and PTB7$\mathrm{Th}: \mathrm{PC}_{71} \mathrm{BM}$ are the opposite. Their temperature dependence of miscibility $/ \chi$ also varies substantially. Furthermore, all the polymers used are high performing for their respective bandgap and class of materials with significant interest to the research community.

PffBT4T- $\mathrm{C}_{9} \mathrm{C}_{13}: \mathrm{PC}_{71} \mathrm{BM}$ is used as the primary model system. PffBT4T- $\mathrm{C}_{9} \mathrm{C}_{13}$ is of high interest for photovoltaic applications due to its benchmark power conversion efficiency it can reach. ${ }^{27}$ Visible light microscopy images acquired of the PffBT4T$\mathrm{C}_{9} \mathrm{C}_{13}: \mathrm{PC}_{71} \mathrm{BM}$ blend annealed at $240^{\circ} \mathrm{C}$ are displayed in Figure 3 , which exhibits the micrometer-scale fullerene crystals that are phase separated out of the blend over time. From the images, 

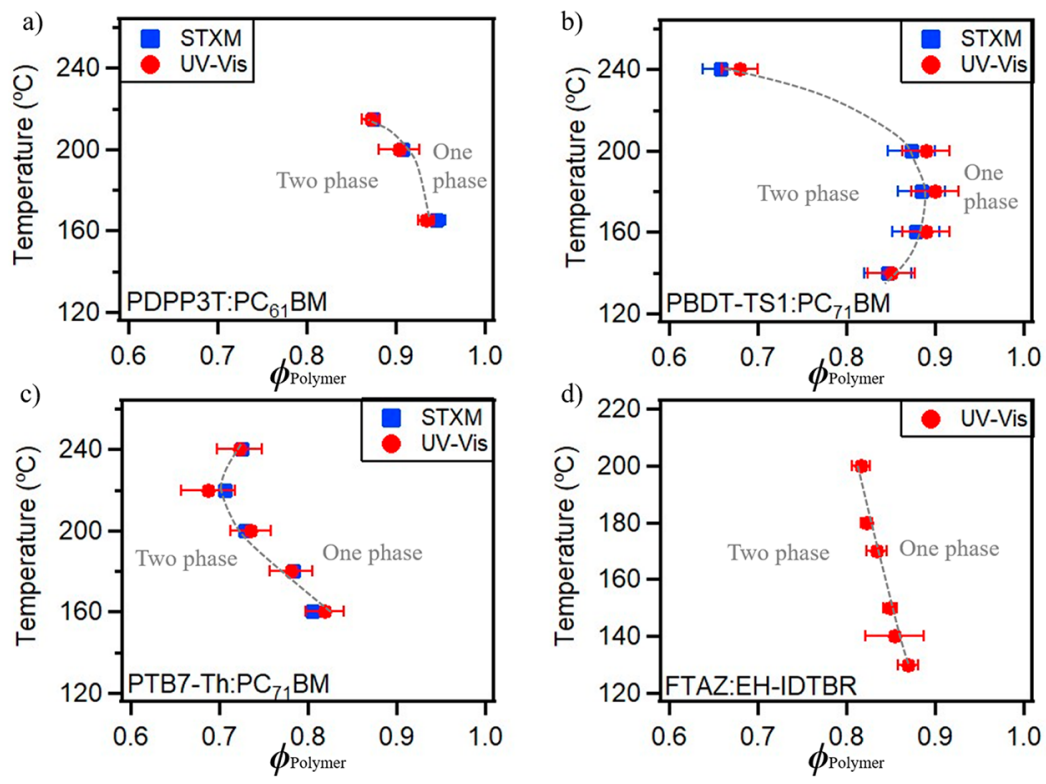

Figure 5. Plots of temperature dependent liquidus and uncorrected liquidus miscibility measured by both the UV-vis absorption method and STXM method for UV-vis absorption spectra of (a) 6:4 (w/w) PDPP3T:PC 61 BM, (b) 6:4 (w/w) PBDT-TS1:PC ${ }_{71}$ BM, and (c) 1:1 (w/w) PTB7$\mathrm{Th}: \mathrm{PC}_{71} \mathrm{BM}$, blends annealed at different temperatures, and the plot of temperature dependent liquidus liquidus miscibility measured by the UV-vis methodology for (d) 7:3 (w/w) FTAZ:EH-IDTBR annealed at different temperatures. The liquidus line separating one phase and two phase regions are conceptual and not fits to a model or theory. The polymer crystals in PDPP3T are not considered and provide likely only a very small perturbation.

during extensive thermal annealing, PCBM in the blends in excess of the liquidus composition will be driven to diffuse out of the mixed phases to form large crystals many micrometers in size, as observed in Figure 3a. Surrounding those crystals, PCBM depletion regions can be observed during the early stages of this process (the light regions in Figure $3 a-3 c) .{ }^{28}$ As a result, the concentration of PCBM in the mixed amorphous phases is reduced, and small phase-separated PCBM domains are eliminated. PCBM depletion regions grow with annealing time until depletion fronts disappear completely (Figure 3d) when (local) thermodynamic equilibrium has been reached. The final, thermodynamic residual PCBM concentration in the amorphous volume fraction of PffBT4T- $\mathrm{C}_{9} \mathrm{C}_{13}$ is determined by the liquidus miscibility between the polymer and the fullerene. In this thermodynamic local equilibrium state (Figure 3e), fullerene crystals are embedded in a uniform matrix without depletion gradients. True equilibrium would be reached if the fullerene had been drawn into a single PCBM crystal. The differences between true and local equilibrium are neglectable for the purpose here. The fullerene volume fraction in the uniform matrix is quantified by the liquidus miscibility once the crystalline volume fraction is taken into consideration. ${ }^{16}$

Figure $4 a-4 c$ shows the transmitted VLM images of the PffBT4T- $\mathrm{C}_{9} \mathrm{C}_{13}: \mathrm{PC}_{71} \mathrm{BM}$ films at thermodynamic equilibrium state after annealing at $160{ }^{\circ} \mathrm{C}, 180{ }^{\circ} \mathrm{C}$, and $200{ }^{\circ} \mathrm{C}$. It is interesting to notice that the fullerene crystals formed during thermal annealing at different temperatures are observed with different shapes and different density, which is of no consequence for our measurements. The absorption spectra of the blend films annealed at different temperatures are shown in Figure 4d. The main difference between the spectra is at low wavelengths below $600 \mathrm{~nm}$, attributable to the much higher absorbance of $\mathrm{PC}_{71} \mathrm{BM}$ than that of PffBT4T-C $\mathrm{C}_{9} \mathrm{C}_{13}$ at low wavelengths as shown in Figure S1. It is also an indication of the different composition of $\mathrm{PC}_{71} \mathrm{BM}$ in the mixed amorphous phase in those measured films. The UV-vis absorption spectra of the annealed PffBT4T-
$\mathrm{C}_{9} \mathrm{C}_{13}: \mathrm{PC}_{71} \mathrm{BM}$ films are then fitted with a linear superposition of the reference spectra acquired from the neat films. Figure $4 \mathrm{f}$ displays the resulting average residual PCBM volume fractions, which is also called the uncorrected liquidus miscibility since the possible pure polymer phase depending on the degree of crystallinity of the polymer has not yet been normalized out.

To crosscheck the validity of the fitted uncorrected liquidus miscibility, scanning transmission X-ray microscopy (STXM) measurement is used as a calibration tool that can avoid the PCBM crystals and measure only between crystals. STXM is well-established and the most used tool to measure the (uncorrected) liquidus miscibility in OPV systems. ${ }^{26}$ To provide context, we briefly delineate the basics of the STXM miscibility measurement methodology. In STXM, an incident soft X-ray beam can be focused by a zone plate onto a small spot (tens of nanometers) on a sample and the transmitted near-edge X-ray absorption fine structure (NEXAFS) spectra are recorded by a detector as a function of the sample position and energy, for example by repeatedly scanning across a certain line in the sample while the energies are varied near the absorption edge. It can differentiate organic materials with high compositional sensitivity and, consequently, is capable of quantifying the composition of the mixed matrix in polymer:fullerene blend films. After the thermodynamic equilibrium state at a certain temperature is reached (Figure 3e), a line scan between and across $\mathrm{PC}_{71} \mathrm{BM}$ crystals can be measured with the assistance of $\mathrm{X}$ ray microscopy. When NEXAFS spectra are also acquired on a neat polymer film and a neat fullerene film, the spectrum of the blend sample at the PCBM depletion region can be fitted with a linear combination of the neat reference spectrum of the polymer and the fullerene, quantifying the uncorrected liquidus miscibility at this temperature. Here, the very same PffBT4T$\mathrm{C}_{9} \mathrm{C}_{13}: \mathrm{PC}_{71} \mathrm{BM}$ blend films used in the UV-vis methodology are characterized by the STXM measurement. The measured NEXAFS spectra are fitted according to the previously established protocol ${ }^{26}$ (see Figure S1 for the fitting details). 

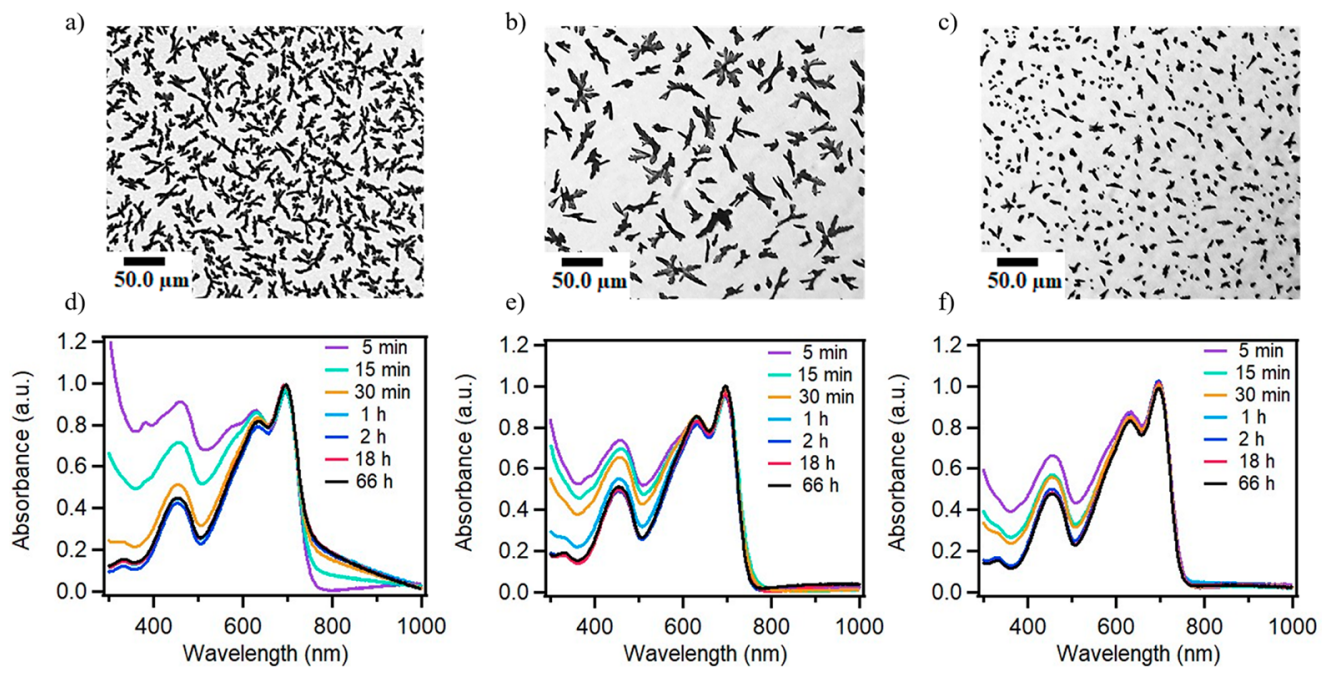

Figure 6. Transmission VLM images of phase-separated blends of (a) 1:1 (w/w) PffBT4T- $\mathrm{C}_{9} \mathrm{C}_{13}: \mathrm{PC}_{71} \mathrm{BM},(\mathrm{b})$ 6:4 (w/w) PffBT4T-C $\mathrm{C}_{13}: \mathrm{PC}_{71} \mathrm{BM}$, and (c) 7:3 (w/w) PffBT4T- $\mathrm{C}_{9} \mathrm{C}_{13}: \mathrm{PC}_{71} \mathrm{BM}$ annealed at $200{ }^{\circ} \mathrm{C}$ for $66 \mathrm{~h}$ and UV-vis absorption spectra at different annealing time for $(\mathrm{d}) 1: 1(\mathrm{w} / \mathrm{w})$ PffBT4T- $\mathrm{C}_{9} \mathrm{C}_{13}: \mathrm{PC}_{71} \mathrm{BM}$, (e) 6:4 (w/w) PffBT4T-C $\mathrm{C}_{13}: \mathrm{PC}_{71} \mathrm{BM}$, and (f) 7:3 (w/w) PffBT4T-C $\mathrm{C}_{13}: \mathrm{PC}_{71} \mathrm{BM}$ annealed at $200{ }^{\circ} \mathrm{C}$.

The resulting average residual fullerene volume fractions in the PCBM-depleted regions at different temperatures are overplotted in Figure 4f. The results from these two methods are consistent with each other well within the error bars of each measurement.

The main difference between the UV-vis methodology and STXM methodology lies in the different beam sizes of the UVvis absorption spectroscopy and STXM used to acquire the spectra, which are hundreds of micrometers and tens of nanometers, respectively. The beam size of a UV-vis spectroscopy is so large that the measured area covers both the mixed amorphous phases and PCBM crystals for the thermally annealed blend films. It seems counterintuitive to quantify the composition of the mixed amorphous phases without enough spatial resolution. However, in an absorption experiment in transmission of any kind, the transmitted flux is subject to an exponential absorption. In samples with large variations in optical density and thicknesses that are larger than those corresponding the linearized region of the exponential absorption, spectral distortions such as absorption saturation occurs as the majority of the signal is from the most transparent regions. Here, we make use of strong absorption saturation effects, and it is ultimately proven that the high absorbance of the large and thick PCBM crystals provides a negligible signal compared with that of the mixed amorphous phases. The error introduced by the crystals is small enough to be neglected for the reason that the final results from these two methodologies match with each other.

After successfully characterizing the uncorrected liquidus miscibility of PffBT4T- $\mathrm{C}_{9} \mathrm{C}_{13}: \mathrm{PC}_{71} \mathrm{BM}$, the UV-vis methodology is extended to three additional polymer:fullerene systems: PDPP3T:PC ${ }_{61}$ BM, PBDT-TS1: PC $_{71}$ BM, PTB7-Th:PC ${ }_{71} B M$, and the nonfullerene system FTAZ:EH-IDTBR. For the PDPP3T:PC ${ }_{61} \mathrm{BM}$ system, PDPP3T is also a semicrystalline polymer similar to PffBT4T- $\mathrm{C}_{9} \mathrm{C}_{13}$ while $\mathrm{PC}_{61} \mathrm{BM}$ is used as the acceptor. The uncorrected liquidus miscibility of the PDPP3T:PC ${ }_{61} \mathrm{BM}$ blend is quantified with the same protocol (details in Figure S2) described above by the UV-vis methodology, showing good agreement with the results from STXM method within the error bars too (Figure 5a). PBDT-TS1 is a common amorphous polymer different from the PffBT4T-
$\mathrm{C}_{9} \mathrm{C}_{13}$. Hence, the liquidus for PBDT-TS1:PC ${ }_{71} \mathrm{BM}$ is measured directly without having to correct for a crystalline volume fraction. The VLM images (Figure S3) show that the PCBM in the PBDT-TS1: PC $_{71} B M$ blend films are also phase-separated to form micrometer-scale fullerene crystals and, consequently, be qualified to quantify the liquidus miscibility with the UV-vis methodology. After fitting the spectra of annealed blend films to the corresponding reference spectra with both UV-vis and STXM methodologies, the results are displayed in Figure $5 b$. They again match well with each other, illustrating the reliability of the UV-vis methodology. For the PTB7-Th:PC ${ }_{71} \mathrm{BM}$ system, PTB7-Th is in the same family with PBDT-TS1. Although it has a similar structure with PBDT-TS1, shapes of the PCBM crystals formed during the thermal annealing are totally different as shown in the VLM images (Figure S4) of the PTB7-Th:PC ${ }_{71} B M$ films at thermodynamic equilibrium state at different annealing temperatures. Quantifying the liquidus miscibility gives a good agreement between both methodologies (Figure 5c). Remarkably, its liquidus has an opposite trend and thermal dependence compared to the PBDT-TS1:PC ${ }_{71} \mathrm{BM}$ system. Neither system seems to be a pure LCST (lower critical solution temperature) or a UCST (upper critical solution temperature) system, which will be discussed later. FTAZ:EH-IDTBR is a nonfullerene polymer:small molecule system. The VLM images (Figure S5) indicate that the micrometer-scale EH-IDTBR crystals can also be generated in the blend films after sufficiently thermal annealing, suggesting that it is eligible to apply this UV-vis method to FTAZ:EH-IDTBR system to quantify the liquidus miscibility. When the spectra of FTAZ:EH-IDTBR blend films annealed at various temperatures are fitted with the reference spectra, the fitted spectra match well with the corresponding measured spectra (Figure S5), demonstrating that this UV-vis methodology should work for nonfullerene polymer:small molecule systems as well and that the methodology can very likely be extended to the many nonfullerene acceptors currently under development. ${ }^{29}$ The results of the liquidus miscibility between FTAZ and EH-IDTBR are shown in Figure 5d. The results from these five different systems demonstrate that the proposed UVvis absorption method is valid and generally applicable to measure the liquidus miscibility of polymer solar cell blends. 
Regarding the $\mathrm{D} / \mathrm{A}$ ratio used in the experiments, it is necessary to demonstrate its impact on the final results as it impacts the density, thickness and volume fraction of the small molecule crystals. Taken as an example, PffBT4T$\mathrm{C}_{9} \mathrm{C}_{13}: \mathrm{PC}_{71} \mathrm{BM}$ blends are made with three different $\mathrm{D} / \mathrm{A}$ weight ratio $(1: 1,6: 4,7: 3)$ and fitted with their corresponding reference spectra, respectively. With increasing D/A ratio, the density and size of PCBM crystals are reduced (Figure 6), and thus the parasitic scattering of long-wavelength light ${ }^{30}$ below the absorption gap due to the crystals reduces, indicated by the flat feature above $800 \mathrm{~nm}$ in the UV-vis absorption spectra shown in Figure 6. After the long-term thermal annealing at the same temperature, the PffBT4T- $\mathrm{C}_{9} \mathrm{C}_{13}: \mathrm{PC}_{71} \mathrm{BM}$ blends with different $\mathrm{D} / \mathrm{A}$ ratios yield almost the same uncorrected liquidus miscibility, proving that the liquidus miscibility is intrinsic to the material system, regardless of PCBM loading. Furthermore, and most importantly, it reconfirms that the PCBM crystals do not materially impact the measurements of the composition of the thin matrix areas between them. But the uncertainty can be reduced with increased D/A ratio. Specifically, the calculated uncorrected liquidus miscibility for $1: 1$ (w/w) PffBT4T$\mathrm{C}_{9} \mathrm{C}_{13}: \mathrm{PC}_{71} \mathrm{BM}$ blends annealed at $200{ }^{\circ} \mathrm{C}$ is $4.67 \pm 2.82 \%$, for 6:4 (w/w) PffBT4T- $\mathrm{C}_{9} \mathrm{C}_{13}: \mathrm{PC}_{71} \mathrm{BM}$ blends annealed at $200^{\circ} \mathrm{C}$ is $4.06 \pm 1.68 \%$ and for $7: 3(\mathrm{w} / \mathrm{w})$ PffBT4T- $\mathrm{C}_{9} \mathrm{C}_{13}: \mathrm{PC}_{71} \mathrm{BM}$ blends annealed at $200{ }^{\circ} \mathrm{C}$ is $3.94 \pm 1.31 \%$ (details in Figure S6). The reduced uncertainty may be attributed to the reduced scattering background at long wavelengths. In principle and if needed for high accuracy absolute results rather than relative measurements to asses the temperature dependence, a $\mathrm{D} / \mathrm{A}$ ratio can be determined and used for which the density of the PCBM crystal is very low.

\section{DISCUSSION}

We note that in many application and devices, it is not the liquidus, but the amorphous miscibility gap, that is, the binodal (see Figure 1), that matters the most when considering device performance and processing strategy. ${ }^{14}$ This has been discussed by Ye et al. for the case of OPVs where the crystallization of the small molecule is suppressed, ${ }^{16}$ Ye et al. also outlined a strategy how to derive the amorphous miscibility from the liquidus miscibility by estimating the chemical potential of the small molecule crystals. This can be achieved with the use of differential scanning calorimetry (DSC) measurements and analyzing the results using phenomenological and theoretical thermodynamic relationships. For amorphous donors, our measurements do not need to be corrected for the crystalline volume fraction and directly yield the liquidus miscibility, and the methodology pioneered by Ye et al. can be used without further corrections. For semicrystalline polymers, our method yields uncorrected liquidus miscibility. In this approach, it is assumed for semicrystalline donors that the polymer crystals have negligible interactions with the small molecule and the measurements are dominated by the amorphous volume fraction. If cocrystals are formed, the results might have be interpreted in terms of a solidus phase transition. The uncorrected data can be converted to liquidus miscibility by normalizing for the volume fraction of the crystalline polymer after determining the degree of crystallinity of the polymer in the blends by a variety of methods. ${ }^{31-33}$ It is also possible that reasonable estimates can be made about the crystallinity from relative measurements and comparisons to benchmark polymers such as $\mathrm{P} 3 \mathrm{HT}$, which have a degree of crystallinity of $30-50 \% .{ }^{31}$ The thus deduced amorphous miscibility and binodal could then be parametrized and modeled using a number of approaches, a topic outside the scope of the current work, but worth considering to understand its eventual reach.

There are generally two main types of phase behavior in T- $\phi$ phase diagrams: ${ }^{34,35}$ lower critical solution temperature (LCST) and upper critical solution temperature (UCST). In LCST, the mixture is miscible at lower temperature and immiscible at higher temperature while it is the opposite in UCST. In the simplest approximation, the $\chi-\phi$ phase diagram can be simulated from and parametrized by the Flory-Huggins solution theory, ${ }^{16}$ and an effective $\chi(T)$ can be deduced after combining the $T-\phi$ and $\chi-\phi$ phase diagrams. Generally, $\chi$ is inversely proportional to the absolute temperature $(T),{ }^{36}$ that is, $\chi=A+B / T$, where $A$ and $B$ are temperature-independent constants and $T$ is the absolute temperature. We note that most organic materials system exhibit UCST behavior and LCST is relatively rare. ${ }^{37-39}$ In contrast, prior STXM work on a number of semiconducting systems has shown that an unusually high fraction of 3 out of 9 systems showed LCST-type behavior. ${ }^{16}$ However, these conclusions were based on only three temperatures. Here, due to extension to more temperatures, we are able to observe even more complex behavior and it is interesting to note that there is a mixture of LCST and UCST in the two amorphous systems: PBDTTS1:PC $\mathrm{P}_{71} \mathrm{BM}$ and PTB7-Th:PC $\mathrm{P}_{71} \mathrm{BM}$ where $\chi=A+B / T$ is not applicable anymore. The $2 \mathrm{D}$ patterns (Figure S7) of GIWAXS (grazing incident wide-angle X-ray scattering) for thermally annealed PBDT-TS1: $\mathrm{PC}_{71} \mathrm{BM}$ films show that only the $\mathrm{PC}_{71} \mathrm{BM}$ is crystallized and tends to be more oriented at high temperatures while the PBDT-TS1 stays amorphous after annealing, suggesting the complicated phase diagram of the PBDTTS1:PC $\mathrm{P}_{71} \mathrm{BM}$ is the result of the amorphous-amorphous interactions. This complicated phase diagram is likely a result of a complex temperature dependence of the entropic contributions related to the differential volume changes and asymmetric thermal expansion as a function of temperature. This unusual behavior might be attributable to the complex molecular rearrangement of the materials at different temperatures. We also note that LCST systems are often associated with strong directional bonds such as hydrogen bonds between the two components of a blend. These attractive bonds lead to mixing at low temperatures and need to be broken first at higher temperatures before the system can phase separate. We note that many semiconducting materials have very dissimilar moieties with a polar/polarizable backbone and very nonpolar side chains. This might lead to directional interactions similar to hydrogen bonds. These complicated interactions and thus currently unpredictable phase diagrams emphasize the necessity of the measurement of liquidus and subsequent binodal to optimize the device performance and stability as discussed by Ye at al. in more details.

The quantitative relation between $\chi_{\text {aa }}$, related to the amorphous miscibility, and device fill factor has been established in a number of PSC systems. ${ }^{14}$ In general, the high amorphous miscibility tends to induce less pure domains and thus increase charge recombination while excessively low amorphous miscibility can result in pure donor or acceptor aggregations where charges might be trapped for the lack of sufficient percolation pathways. An amorphous miscibility that is too low corresponds to a high thermodynamic limit of the domain purity in the mixed amorphous phase which may result in the spontaneous phase demixing of quenched morphologies and burn-in degradation. For example, PffBT4T- $\mathrm{C}_{9} \mathrm{C}_{13}: \mathrm{PC}_{71} \mathrm{BM}$, which shows a very low uncorrected liquidus miscibility beyond 
the percolation threshold, is reported to have a decrease in $J_{\mathrm{sc}}$ of over $10 \%$ for the devices stored even in the dark for 5 days due to the burn-in degradation as the system evolves from a quenched state toward the metastable equilibrium of the binodal. ${ }^{16}$

We have demonstrated that the proposed UV-vis methodology can be successfully applied to polymer:fullerene and polymer:nonfullerene small molecule systems to measure the (uncorrected) liquidus miscibility and would like to briefly discuss the possible extension to polymer-polymer and smallmolecule:small-molecule systems. The latter should be straightforward, as long as one of the components crystallizes. If neither component crystallizes, then a method with sufficient spatial resolution is required to interrogate the individual domains that are both possibly in a mixed state. Since it is too difficult for polymer blends to phase separate and form the micrometer-size, thick pure crystals of a single kind of polymer during thermal annealing, it will be impossible to apply this UV-vis methodology to all-polymer systems. Bilayer interdiffusion experiment by SIMS or reflectivity methods might then be the most appropriate method.

\section{CONCLUSION}

In summary, a novel and widely accessible method based on the combination of visible light microscopy and a UV-vis absorption spectroscopy has been successfully developed to quantify the temperature-dependent (uncorrected) liquidus miscibility between the polymer donor and small molecule acceptor. This UV-vis method has shown beneficial characteristics such as wide accessibility, easy operation, general applicability and good reliability. Consequently, the UV-vis method shows promise of replacing STXM and becoming a common method in characterizing the uncorrected liquidus miscibility. Nowadays, hundreds of nonfullerene small molecule acceptors are synthesized to replace fullerene acceptors ${ }^{40}$ and obtain the record power conversion efficiency up to $>14 \%{ }^{41}$ As demonstrated herein, this UV-vis method is also applicable for these nonfullerene systems to understand the impact of the miscibility between the donor and the nonfullerene acceptor, which can help predict the morphology of a certain system. It will be instructive in screening out the promising materials combinations when new materials are synthesized and constructive by optimizing the processing strategies such as the processing temperature for a system before laborious trial-and-error. Additionally, the ease and wide accessibility of this developed method can assist to make the concept of liquidus miscibility more extensively utilized and further develop thermodynamic studies. Since the miscibility between different materials is a general parameter significant in determining the property in many different aspects, this UV-vis methodology has the potential to be applied in other research fields beyond organic solar cells, such as organic light-emitting diodes (OLED), organic field-effect transistors (OFET), ferroelectric devices and organic thermoelectrics and other two component materials systems with partial miscibility.

\section{ASSOCIATED CONTENT}

\section{S Supporting Information}

The Supporting Information is available free of charge on the ACS Publications website at DOI: 10.1021/acs.chemmater.8b00889.

Experimental details on the film fabrication, VLM images, UV-vis spectra of aggressively thermally annealed films, the fitted UV-vis spectra, STXM results, and GIWAXS patterns (PDF)

\section{AUTHOR INFORMATION}

\section{Corresponding Author}

*E-mail: harald_ade@ncsu.edu. ORCID

Long Ye: 0000-0002-5884-0083

Sunsun Li: 0000-0003-3581-8358

Jianhui Hou: 0000-0002-2105-6922

Harald Ade: 0000-0002-7871-1158

\section{Author Contributions}

Z.P. and X.J. contributed equally to this work.

\section{Notes}

The authors declare no competing financial interest.

\section{ACKNOWLEDGMENTS}

Work by NCSU was supported by ONR grant N0014-15-1-2322, N0014-17-1-2204, and UNC-GA ROI grant. STXM data were acquired at beamline 5.3.2.2 $2^{25}$ at the Advanced Light Source, which is supported by the Director, Office of Science, Office of Basic Energy Sciences, of the U.S. Department of Energy under Contract No. DE-AC02-05CH11231. J.J.R. and W.Y. were supported by the NSF grant CBET-1639429.

\section{REFERENCES}

(1) Brédas, J. L.; Norton, J. E.; Cornil, J.; Coropceanu, V. Molecular Understanding of Organic Solar Cells: The Challenges. Acc. Chem. Res. 2009, 42, 1691-1699.

(2) Abbaszadeh, D.; Kunz, A.; Wetzelaer, G. A. H.; Michels, J. J.; Crăciun, N. I.; Koynov, K.; Lieberwirth, I.; Blom, P. W. M. Elimination of charge carrier trapping in diluted semiconductors. Nat. Mater. 2016, 15, 628-633.

(3) Nikolka, M.; Nasrallah, I.; Rose, B.; Ravva, M. K.; Broch, K.; Sadhanala, A.; Harkin, D.; Charmet, J.; Hurhangee, M.; Brown, A.; Illig, S.; Too, P.; Jongman, J.; McCulloch, I.; Bredas, J. L.; Sirringhaus, H. High operational and environmental stability of high-mobility conjugated polymer field-effect transistors through the use of molecular additives. Nat. Mater. 2017, 16, 356-362.

(4) Katsouras, I.; Asadi, K.; Li, M.; van Driel, T. B.; Kjaer, K. S.; Zhao, D.; Lenz, T.; Gu, Y.; Blom, P. W. M.; Damjanovic, D.; Nielsen, M. M.; de Leeuw, D. M. The negative piezoelectric effect of the ferroelectric polymer poly(vinylidene fluoride). Nat. Mater. 2016, 15, 78-84.

(5) Li, W. T.; Yang, L. Q.; Tumbleston, J. R.; Yan, L.; Ade, H.; You, W. Controlling Molecular Weight of a High Efficiency Donor-Acceptor Conjugated Polymer and Understanding Its Significant Impact on Photovoltaic Properties. Adv. Mater. 2014, 26, 4456-4462.

(6) Collins, B. A.; Gann, E.; Guignard, L.; He, X.; McNeill, C. R.; Ade, H. Molecular Miscibility of Polymer-Fullerene Blends. J. Phys. Chem. Lett. 2010, 1, 3160-3166.

(7) Treat, N. D.; Varotto, A.; Takacs, C. J.; Batara, N.; Al-Hashimi, M.; Heeney, M. J.; Heeger, A. J.; Wudl, F.; Hawker, C. J.; Chabinyc, M. L. Polymer-Fullerene Miscibility: A Metric for Screening New Materials for High-Performance Organic Solar Cells. J. Am. Chem. Soc. 2012, 134, $15869-15879$.

(8) Bartelt, J. A.; Beiley, Z. M.; Hoke, E. T.; Mateker, W. R.; Douglas, J. D.; Collins, B. A.; Tumbleston, J. R.; Graham, K. R.; Amassian, A.; Ade, H.; Fréchet, J. M. J.; Toney, M. F.; McGehee, M. D. The Importance of Fullerene Percolation in the Mixed Regions of Polymer-Fullerene Bulk Heterojunction Solar Cells. Adv. Energy Mater. 2013, 3, 364-374.

(9) Ye, L.; Zhao, W.; Li, S.; Mukherjee, S.; Carpenter, J. H.; Awartani, O.; Jiao, X.; Hou, J.; Ade, H. High-Efficiency Nonfullerene Organic Solar Cells: Critical Factors that Affect Complex Multi-Length Scale Morphology and Device Performance. Adv. Energy Mater. 2017, 7, 1602000 . 
(10) Bin, H.; Yang, Y.; Zhang, Z.; Ye, L.; Ghasemi, M.; Chen, S.; Zhang, Y.; Zhang, C.; Sun, C.; Xue, L.; Yang, C.; Ade, H.; Li, Y. 9.73\% Efficiency Nonfullerene All Organic Small Molecule Solar Cells with AbsorptionComplementary Donor and Acceptor. J. Am. Chem. Soc. 2017, 139, 5085-5094.

(11) Ma, W.; Tumbleston, J. R.; Wang, M.; Gann, E.; Huang, F.; Ade, H. Domain Purity, Miscibility, and Molecular Orientation at Donor/ Acceptor Interfaces in High Performance Organic Solar Cells: Paths to Further Improvement. Adv. Energy Mater. 2013, 3, 864-872.

(12) Mukherjee, S.; Proctor, C. M.; Bazan, G. C.; Nguyen, T.-Q.; Ade, $\mathrm{H}$. Significance of Average Domain Purity and Mixed Domains on the Photovoltaic Performance of High-Efficiency Solution-Processed SmallMolecule BHJ Solar Cells. Adv. Energy Mater. 2015, 5, 1500877.

(13) Mukherjee, S.; Proctor, C. M.; Tumbleston, J. R.; Bazan, G. C.; Nguyen, T.-Q.; Ade, H. Importance of Domain Purity and Molecular Packing in Efficient Solution-Processed Small-Molecule Solar Cells. Adv. Mater. 2015, 27, 1105-1111.

(14) Ye, L.; Hu, H.; Ghasemi, M.; Wang, T.; Collins, B. A.; Kim, J. H.; Jiang, K.; Carpenter, J. H.; Li, H.; Li, Z.; McAfee, T.; Zhao, J.; Chen, X.; Lai, J. L. Y.; Ma, T.; Bredas, J. L.; Yan, H.; Ade, H. Quantitative relations between interaction parameter, miscibility and function in organic solar cells. Nat. Mater. 2018, 17, 253-260.

(15) Tumbleston, J. R.; Yang, L. Q.; You, W.; Ade, H. Morphology linked to miscibility in highly amorphous semi-conducting polymer/ fullerene blends. Polymer 2014, 55, 4884-4889.

(16) Ye, L.; Collins, B. A.; Jiao, X.; Zhao, J.; Yan, H.; Ade, H. Miscibility-Function Relations in Organic Solar Cells: Significance of Optimal Miscibility in Relation to Percolation. Adv. Energy Mater. 2018, 8, 1703058 .

(17) Bartelt, J. A.; Beiley, Z. M.; Hoke, E. T.; Mateker, W. R.; Douglas, J. D.; Collins, B. A.; Tumbleston, J. R.; Graham, K. R.; Amassian, A.; Ade, H.; Frechet, J. M. J.; Toney, M. F.; McGehee, M. D. The Importance of Fullerene Percolation in the Mixed Regions of Polymer-Fullerene Bulk Heterojunction Solar Cells. Adv. Energy Mater. 2013, 3, 364-374.

(18) Collins, B. A.; Li, Z.; McNeill, C. R.; Ade, H. Fullerene-Dependent Miscibility in the Silole-Containing Copolymer PSBTBT-08. Macromolecules 2011, 44, 9747-9751.

(19) Hartmeier, B. F.; Brady, M. A.; Treat, N. D.; Robb, M. J.; Mates, T. E.; Hexemer, A.; Wang, C.; Hawker, C. J.; Kramer, E. J.; Chabinyc, M. L. Significance of miscibility in multidonor bulk heterojunction solar cells. J. Polym. Sci., Part B: Polym. Phys. 2016, 54, 237-246.

(20) Vakhshouri, K.; Kozub, D. R.; Wang, C.; Salleo, A.; Gomez, E. D. Effect of miscibility and percolation on electron transport in amorphous poly(3-hexylthiophene)/phenyl-c61-butyric acid methyl ester blends. Phys. Rev. Lett. 2012, 108, 026601.

(21) Treat, N. D.; Brady, M. A.; Smith, G.; Toney, M. F.; Kramer, E. J.; Hawker, C. J.; Chabinyc, M. L. Interdiffusion of PCBM and P3HT Reveals Miscibility in a Photovoltaically Active Blend. Adv. Energy Mater. 2011, 1, 82-89.

(22) Kozub, D. R.; Vakhshouri, K.; Orme, L. M.; Wang, C.; Hexemer, A.; Gomez, E. D. Polymer Crystallization of Partially Miscible Polythiophene/Fullerene Mixtures Controls Morphology. Macromolecules 2011, 44, 5722-5726.

(23) Ye, L.; Zhang, S.; Zhao, W.; Yao, H.; Hou, J. Highly Efficient 2DConjugated Benzodithiophene-Based Photovoltaic Polymer with Linear Alkylthio Side Chain. Chem. Mater. 2014, 26, 3603-3605.

(24) Price, S. C.; Stuart, A. C.; Yang, L.; Zhou, H.; You, W. Fluorine substituted conjugated polymer of medium band gap yields $7 \%$ efficiency in polymer-fullerene solar cells. J. Am. Chem. Soc. 2011, 133, $4625-4631$.

(25) Kilcoyne, A. L. D.; Tyliszczak, T.; Steele, W. F.; Fakra, S.; Hitchcock, P.; Franck, K.; Anderson, E.; Harteneck, B.; Rightor, E. G.; Mitchell, G. E.; Hitchcock, A. P.; Yang, L.; Warwick, T.; Ade, H. Interferometer-controlled scanning transmission X-ray microscopes at the Advanced Light Source. J. Synchrotron Radiat. 2003, 10, 125-136. (26) Collins, B. A.; Ade, H. Quantitative compositional analysis of organic thin films using transmission NEXAFS spectroscopy in an X-ray microscope. J. Electron Spectrosc. Relat. Phenom. 2012, 185, 119-128.
(27) Zhao, J. B.; Li, Y. K.; Yang, G. F.; Jiang, K.; Lin, H. R.; Ade, H.; Ma, W.; Yan, H. Efficient organic solar cells processed from hydrocarbon solvents. Nat. Energy 2016, 1, 15027.

(28) Watts, B.; Belcher, W. J.; Thomsen, L.; Ade, H.; Dastoor, P. C. A Quantitative Study of PCBM Diffusion during Annealing of P3HT: PCBM Blend Films. Macromolecules 2009, 42, 8392-8397.

(29) Zhang, G.; Zhao, J.; Chow, P. C. Y.; Jiang, K.; Zhang, J.; Zhu, Z.; Zhang, J.; Huang, F.; Yan, H. Nonfullerene Acceptor Molecules for Bulk Heterojunction Organic Solar Cells. Chem. Rev. 2018, 118, 3447-3507.

(30) Lindberg, J. D.; Snyder, D. G. Determination of the optical absorption coefficient of powdered materials whose particle size distribution and refractive indices are unknown. Appl. Opt. 1973, 12, 573-578.

(31) Shen, X. B.; Hu, W. G.; Russell, T. P. Measuring the Degree of Crystallinity in Semicrystalline Regioregular Poly(3-hexylthiophene). Macromolecules 2016, 49, 4501-4509.

(32) Balko, J.; Lohwasser, R. H.; Sommer, M.; Thelakkat, M.; ThurnAlbrecht, T. Determination of the Crystallinity of Semicrystalline Poly(3-hexylthiophene) by Means of Wide-Angle X-ray Scattering. Macromolecules 2013, 46, 9642-9651.

(33) Snyder, C. R.; Nieuwendaal, R. C.; DeLongchamp, D. M.; Luscombe, C. K.; Sista, P.; Boyd, S. D. Quantifying Crystallinity in High Molar Mass Poly(3-hexylthiophene). Macromolecules 2014, 47, 39423950.

(34) Bates, F. S. Polymer-polymer phase behavior. Science 1991, 251, 898-905.

(35) Knychała, P.; Timachova, K.; Banaszak, M.; Balsara, N. P. 50th Anniversary Perspective: Phase Behavior of Polymer Solutions and Blends. Macromolecules 2017, 50, 3051-3065.

(36) Russell, T. P.; Hjelm, R. P.; Seeger, P. A. Temperature dependence of the interaction parameter of polystyrene and poly(methyl methacrylate). Macromolecules 1990, 23, 890-893.

(37) Bandrup, J.; Immergut, E. H.; Grulke, E. A. Polymer Handbook; Interscience Publishers: New York, 1966.

(38) Ougizawa, T.; Inoue, T. UCST and LCST Behavior in Polymer Blends and Its Thermodynamic Interpretation. Polym. J. 1986, 18, 521527.

(39) Roth, P. J.; Davis, T. P.; Lowe, A. B. Comparison between the LCST and UCST Transitions of Double Thermoresponsive Diblock Copolymers: Insights into the Behavior of POEGMA in Alcohols. Macromolecules 2012, 45, 3221-3230.

(40) Nielsen, C. B.; Holliday, S.; Chen, H.-Y.; Cryer, S. J.; McCulloch, I. Non-Fullerene Electron Acceptors for Use in Organic Solar Cells. Acc. Chem. Res. 2015, 48, 2803-2812.

(41) Li, S.; Ye, L.; Zhao, W.; Yan, H.; Yang, B.; Liu, D.; Li, W.; Ade, H.; Hou, J. A Wide Band Gap Polymer with a Deep Highest Occupied Molecular Orbital Level Enables 14.2\% Efficiency in Polymer Solar Cells. J. Am. Chem. Soc. 2018, DOI: 10.1021/jacs.8b02695. 